\title{
AVANCES EN EL ESTUdIO DE BIODETERIORO ASOCIADO A PRESENCIA DE LíQUENES EN MATERIALES ARQUEOLÓgICOS Líticos. LA PRIMAVERA (Santa Cruz, Argentina)
}

\author{
LUCIA ANGELICA MAGNIN¹, VIRGINIA LYNCH² y RENATO GRACÍA
}

\begin{abstract}
Summary: Advances in the study of biodeterioration associated to the presence of lichens on archaeological lithic materials. La Primavera (Santa Cruz, Argentina). In this work, the biodeterioration caused by lichens growing on lithic archaeological artifacts from La Primavera was analyzed. The methodology includes observation techniques of low and high magnifications and EDAX analysis. The lichens present were identified and the surfaces of the lithic artifacts where they grow were observed. We identified and analyzed the diversity of species present and recognized four types of bioalterations (micropitting, superficial weathering, internal micro-fractures and superficial micro-fractures). Among the identified morphotypes, which apparently causes the most damage is the crustose endolithic, followed by foliose. The bioalterations registered hinder the observation of the main technological characteristics as well as specific studies on the functionality of the artifacts, what in turn results in information loss. Knowing more in detail how these bioalterations affect the lithic materials is relevant for the archaeological interpretations. Therefore, this work is an important contribution to the study of biodeterioration in lithic artifacts that has not been the subject of specific investigations before.
\end{abstract}

Key words: Biodeterioration, lichens, lithic archaeological artefacts.

Resumen: Con el objetivo de indagar acerca del biodeterioro causado por líquenes creciendo sobre materiales arqueológicos líticos, en este trabajo preliminar se analizaron diferentes artefactos procedentes de la localidad La Primavera. La metodología de análisis incluye técnicas de observación de bajos y altos aumentos y análisis de EDAX. Se identifican los líquenes presentes y se observan las superficies de estos artefactos que constituyen soportes para su crecimiento. Se observó y analizó la diversidad de especies presentes y se reconocieron cuatro tipos de bioalteraciones asociadas (microperforaciones, desagregación superficial de la roca, microfracturaciones internas y microfracturaciones superficiales). Entre los morfotipos identificados, el que al parecer causa mayores daños es el crustoso endolítico, seguido del folioso. Estas bioalteraciones dificultan la observación de los caracteres tecnológicos principales, así como estudios específicos acerca de la funcionalidad del instrumental lítico, lo que produce pérdida de información. Conocer más detalladamente cómo estas bioalteraciones afectan a estos materiales es relevante para las interpretaciones arqueológicas. Por lo tanto, este trabajo es una importante contribución al estudio de biodeterioro sobre artefactos líticos que hasta el momento no ha sido objeto de investigaciones específicas.

Palabras clave: Biodeterioro, líquenes, artefactos arqueológicos líticos.

${ }^{1}$ División Arqueología, FCNyM - Facultad de Ciencias Naturales y Museo, UNLP - Universidad Nacional de La Plata, CONICET - Consejo Nacional de Investigaciones Científicas y Técnicas. Imagnin@fcnym.unlp.edu.ar

${ }^{2}$ División de Arqueología, FCNyM - Facultad de Ciencias Naturales y Museo, UNLP- Universidad Nacional de La Plata, CONICET - Consejo Nacional de Investigaciones Científicas y Técnicas. lynchvirginia@gmail.com

${ }^{3}$ LEMIT-CICPBA, CONICET - Consejo Nacional de Investigaciones Científicas y Técnicas. Garciarenato86@gmail.com 


\section{INTRODUCCIÓN}

Como colonizadores pioneros de rocas, los líquenes pueden crear un microambiente favorable al aumentar la biodisponibilidad de elementos minerales y nutrientes para otras formas de vida, que también contribuyan a la erosión de las rocas y el desarrollo del suelo (García-Rowe \& SaizJiménez, 1991; Harper \& Pendleton, 1993). De acuerdo a su crecimiento y la adhesión al sustrato se puede clasificar a los líquenes en tres formas básicas: crustosos son aquellos que no poseen nunca córtex inferior, se sujetan al sustrato a través de las hifas de la médula o de las del hipotalo, se encuentran en íntimo contacto con el sustrato y son difícilmente separables de él; foliosos son aquellos que tienen el talo con una forma laminar lobulada, con simetría dorsiventral, su relación con el sustrato se hace a través del cortex inferior, mediante un único apéndice central (ombligo) o de varios órganos apendiculares (rizines); y por último, los fruticosos presentan un crecimiento perpendicular a la superficie y se sujetan al sustrato por medio de un disco de adhesión pequeño (Barreno \& Rico, 1984; Büdel \& Scheidegger, 2008). Los crustosos saxícolas se pueden subcategorizar como epilíticos o endolíticos. En los epilíticos, la mayor parte del talo crece sobre la superficie del sustrato rocoso aunque en algunas ocasiones sus hifas también se observaron penetrando la superficie de la roca (Bjelland \& Ekman, 2000; Souza-Egipsy et al., 2002). En cambio, los talos endolíticos crecen dentro del sustrato, aunque según Golubic et al., (1981) algunas especies de líquenes son parcialmente epilíticas y/o endolíticas, mientras que otras atraviesan ambas fases en el curso de su ciclo de vida. Todos los hábitos de líquenes generan cambios sobre sustratos rocosos, pero son los crustosos, por su mayor superficie y grado de adherencia al sustrato, los que producen el mayor número de efectos físicos y químicos. Los efectos físicos comienzan con la penetración de las hifas a través de la porosidad de la roca. Este es uno de los procesos más importantes, dado que contribuye directamente al daño por meteorización física y mecánica de las rocas, y también induce y acelera otras formas de alteraciones físicas. Las especies crustosas pueden causar exfoliación y disgregación de la roca, por la penetración de las hifas en el sustrato. Mientras que las especies foliosas penetran el sustrato solo por sus rizines, que pueden expandirse dentro del mismo causando perforaciones y agrietando la superficie (Chen et al., 2000).

En cuanto a los efectos químicos, en su mayoría se encuentran relacionados con los procesos metabólicos liquénicos. El ácido orgánico secretado por el micobionte de muchos líquenes tiene un rol importante en el deterioro de las rocas, especialmente el ácido oxálico (Camuffo, 1995; Chen et al., 2000). Otras especies, pueden secretar ácidos orgánicos simples como el cítrico, láctico y el glucónico, que también pueden conducir a la erosión de las rocas a través del ataque ácido y su actividad quelante (Chen et al., 2000). Además, la disolución del $\mathrm{CO}_{2}$ respiratorio en agua resulta en la generación de ácidos carbónicos, estos bajan el $\mathrm{pH}$ circundante al talo produciendo un microambiente propicio para los procesos de solubilización, que contribuyen a la degradación química (Seaward et al.1989; Wierzchos \& Ascaso, 1996). Asimismo, otras daños en la roca pueden ocasionarse en la interfaz liquen-roca, por la presión ejercida de los cristales en formación como consecuencia de la cristalización secundaria de sales (oxalatos) (Sand, 1997).

Ambos tipos de procesos, mecánicos y químicos no se dan de forma aislada, sino que funcionan de una forma conjunta, produciendo un efecto significativo en la meteorización y jugando un papel muy importante en la degradación de las rocas y formación del suelo. En relación con lo antes dicho, los líquenes son capaces de crecer casi sobre cualquier tipo de sustrato, por lo cual los materiales arqueológicos en superficie tienen potencial para ser colonizados por líquenes y por lo tanto, también están sujetos a estos procesos de deterioro. Si bien se ha registrado en trabajos arqueológicos frecuentemente la presencia de estos organismos, hasta el momento existen escasos estudios acerca de los efectos generados por la acción liquénica en artefactos líticos arqueológicos (Benedict, 2009; Borrazzo, 2011).

La localidad arqueológica La Primavera se localiza en el Macizo Central del Deseado, provincia de Santa Cruz. Está caracterizada por ambientes serranos y de mesetas volcánicas de alturas variables entre los 500 y $1000 \mathrm{msnm}$. En algunos sectores los mantos basálticos protegen a las unidades sedimentarias inferiores, más fácilmente erosionables, que toman la forma de serranías y en ocasiones son cortadas por profundos cañadones, como el Cañadón 
La Primavera (Fig. 1). Completan las unidades geomorfológicas las terrazas constituidas por niveles de pedimentos y las planicies aluviales (Gómez \& Magnin, 2008). La Meseta Central de Santa Cruz corresponde con el territorio fitogeográfico denominado Provincia Patagónica que a su vez se encuentra dentro del Dominio Andino-Patagónico. En el área de estudio la vegetación dominante es la estepa arbustiva y sub-arbustiva, con sectores de estepa herbácea, donde abundan las formas extremas de adaptación al viento y a la sequía (Cabrera, 1971). La región presenta un clima templado frío árido de meseta, con temperaturas medias que varían entre 8 y $10^{\circ} \mathrm{C}$ y precipitaciones medias anuales menores a $200 \mathrm{~mm}$ (Soto \& Vázquez, 2000). La circulación atmosférica, asociada al anticiclón del Pacífico, determina la existencia de vientos que varían entre brisas fuertes y vientos fuertes (36 a $55 \mathrm{Km} / \mathrm{h}$ ). La localidad fue ocupada por sociedades cazadoras-recolectoras durante al menos 8400 años $\mathrm{AP}$, según pudo determinarse a partir de evidencia arqueológica asociada a fechados radiocarbónicos obtenidos en sitios localizados en cuevas y a cielo abierto (Magnin, 2010; Miotti et al., 2014). Además de evidencias en sitios estratigráficos, muchos materiales arqueológicos, principalmente artefactos líticos de alta perdurabilidad, se hallaron en la superficie del terreno aislados o formando conjuntos de distintas densidades. Parte de esos artefactos líticos de superficie presentan líquenes (Magnin, 2010; Magnin et al., 2013; García et al., 2015), lo que llevó a la formulación de interrogantes acerca de qué clase de deterioro causan esos organismos sobre los artefactos y si es distinguible del deterioro causado por otros agentes o procesos. Por lo tanto, en este trabajo nos proponemos hacer una aproximación exploratoria para registrar y describir bioalteraciones asociadas a la presencia de líquenes presentes sobre las superficies de artefactos líticos. Asimismo, se indaga sobre la diversidad de estas bioalteraciones teniendo en consideración los tipos morfológicos presentes. El conocimiento de la acción de líquenes sobre estos artefactos de tamaños relativamente pequeños es un área de interés para el estudio de los procesos de formación de sitios arqueológicos que ha sido muy poco explorada.

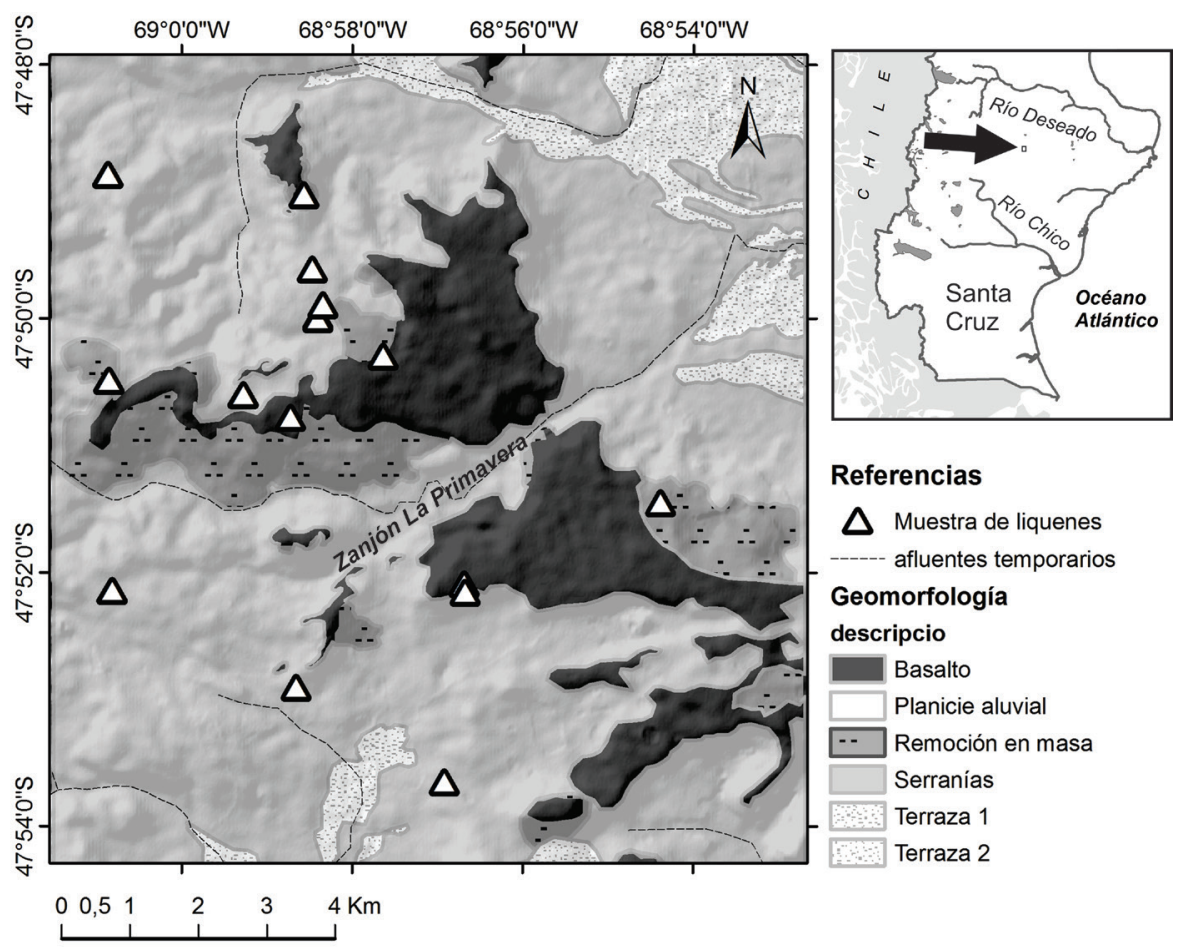

Fig. 1. Localización y geomorfología del área de estudio (Gómez y Magnin, 2008). 


\section{Material y Método}

\section{Muestra analizada}

Los artefactos recolectados en el área de estudio ( $c a . \mathrm{n}=6000)$, fueron observados a ojo desnudo y lupa de mano (10X) para detectar líquenes presentes sobre superficies modificadas tecnológicamente y/o sobre la corteza natural de las rocas (Magnin, 2010; García et al., 2015). De este conjunto, 190 artefactos presentaron líquenes creciendo sobre sus superficies. La muestra analizada en este trabajo está constituida por 21 artefactos y dos muestras de ocre, seleccionados por presentar líquenes con talos bien desarrollados que facilitaron su identificación. Las materias primas utilizadas en su producción son de procedencia local (Magnin et al., 2015). Predominan los sílices de color marrón, amarillo y blanco, con calidades para la talla que van desde regular a excelente; seguidos por riolitas en color marrón y rojo, de calidad buena a mala; calcedonias y xilópalos de color marrón y de excelente calidad. Desde el aspecto tecno-morfológico (Aschero, 1975), la muestra incluye en su mayoría artefactos no formatizados $(n=14)$, dos formatizados (un raspador de filo frontal corto y una muesca) y tres núcleos. Las medidas promedio de las piezas arqueológicas son de $469 \mathrm{~mm}$ de largo, $375 \mathrm{~mm}$ de ancho y $147 \mathrm{~mm}$ de espesor, y pesan en promedio $37,30 \mathrm{~g}$.

\section{Técnicas de observación}

\section{Observación a bajos aumentos}

El crecimiento de los líquenes en relación con las caras de las piezas se registró a ojo desnudo y bajo lupa binocular (10X-63X), indicando si se encontraban en la cara dorsal o ventral del artefacto, o sobre un borde (dorso, talón o superficie de fractura) (Aschero, 1975, Rev.1983). Asimismo se registró si se dispone sobre la corteza (superficie original de la roca) o en sectores desprovistos de esta (superficies modificadas por talla lítica).

Para la identificación de las especies se observó la morfología externa de los talos utilizando una lupa trinocular (Leica S8 APO y Nikon SMZ800 con aumentos de 10 a $63 \mathrm{X}$ ), y se realizaron cortes histológicos y reacciones puntuales con hipoclorito de sodio (C), hidróxido de potasio (K) y luz ultravioleta. A su vez, se seleccionaron cuatro piezas en las cuales se retiró una porción del talo con peróxido de hidrógeno $(10 \%)$ para poder observar la superficie del artefacto buscando posibles alteraciones. Se utilizaron las claves de identificación de Lamb (1958), Nash et al. (1995), Ovstedal \& Lewis-Smith (2001), Nash et al. (2004), Śliwa (2007) y De la Rosa et al. (2012). En esta primera instancia, los líquenes de la muestra observada fueron clasificados según sus morfotipos (crustoso endolítico, crustoso epilítico y folioso) y a nivel de familia, género o especie.

\section{Observación a altos aumentos}

Posteriormente, a partir de un microscopio metalográfico Nikon Epiphoto 200 (50X-500X) bajo campo claro, se realizó el registro de los líquenes teniendo en cuenta su distribución en relación a la microtopografía de la pieza. De acuerdo a esto se consideró su disposición en: sectores altos (en cúspides o salientes del filo); bajos (en negativos de lascados o sectores de fracturas) y homogénea (en sectores altos y bajos).

En los casos de líquenes creciendo sobre los filos, se siguió la metodología de Álvarez (2003), donde se considera su disposición como marginal (aquellos que se extienden menos de $1 / 3$ del diámetro de campo, es decir, menos de 0,33 mm.) y muy profundo (más de $3 / 3$ de diámetro de campo, más de $1 \mathrm{~mm}$.). Asimismo se registró si los líquenes se localizaron sobre aristas (Aschero 1975, Rev.1983).

Una vez removido el talo de cada liquen para dejar al descubierto la superficie del artefacto lítico, se registraron las posibles bioalteraciones presentes. Los tipos de bioalteraciones a registrar incluyen la presencia de desagregación superficial de la roca; microperforaciones, microfracturaciones internas y microfracturaciones superficiales.

La desagregación superficial de la roca se refiere a una modificación extendida que no implica una discontinuidad sobre la superficie rocosa, presenta un aspecto rugoso y de profundidad relativa menor que en el caso de las microperforaciones. A diferencia de las microfisuraciones internas, este tipo de alteración puede ser consecuencia de la fijación del liquen sobre el sustrato (Fig. 2c).

Las microperforaciones son modificaciones puntuales de la roca con un diámetro menor 

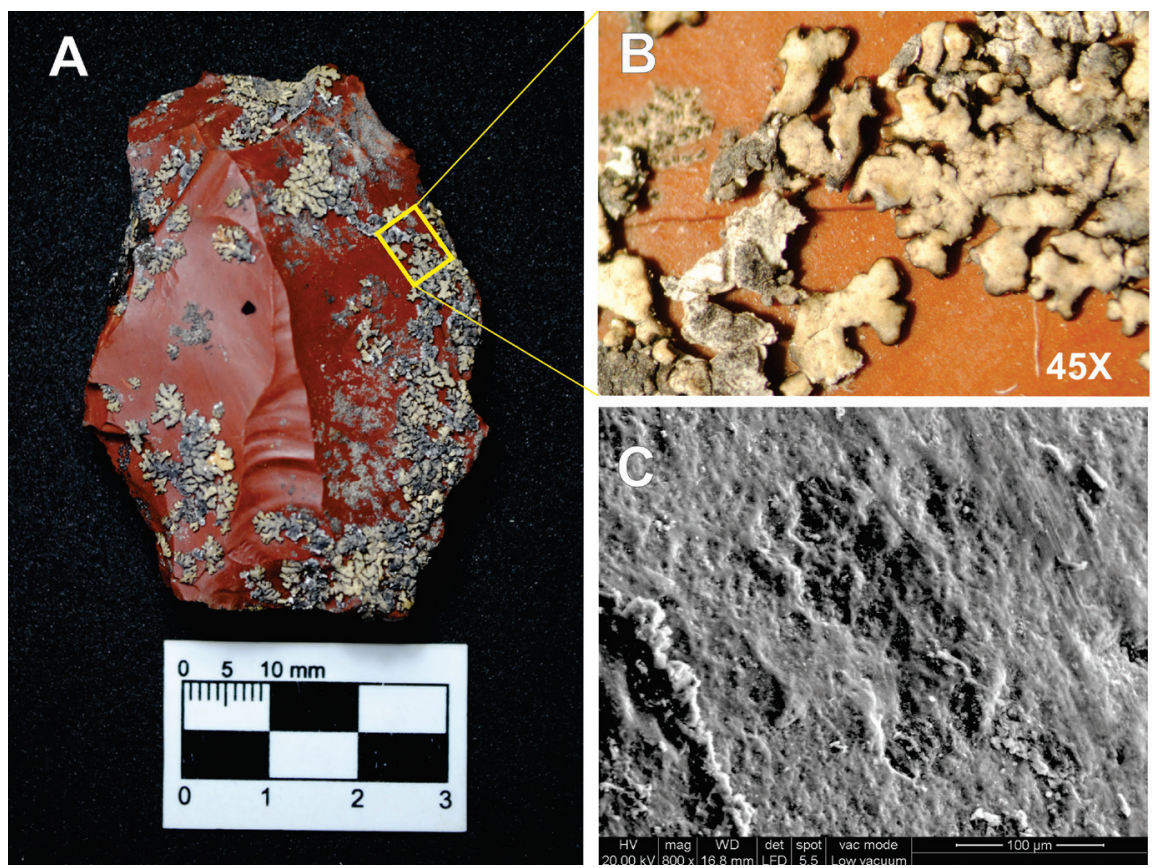

Fig. 2. A: Artefacto B13; B: Liquen folioso (X. xanthomelanea) observado mediante lupa binocular; C: Imagen de MEB de un sector de la pieza donde fue removido el talo donde se observa desagregación superficial.

a $1 \mathrm{~mm}$, de morfología oval a semi-circular y cierta profundidad hacia el interior de la roca. Estas pueden ser producidas durante la fijación de rizines. En cambio en el caso de los líquenes endolíticos se generan como consecuencia de la exposición del cuerpo fructífero (Fig. 3c).

Las microfracturaciones internas son cavidades dispuestas a unos micrones por debajo de la superficie rocosa, al igual que en el caso anterior tienen una morfología ramificada. Estas pueden deberse a la acción físico-química de las hifas durante la penetración de la roca (Fig. 4b).

Las microfracturaciones superficiales son discontinuidades de la superficie rocosa, de morfología ramificada. Su presencia en la superficie de rocas colonizadas por líquenes está asociada a la expansión del talo, proceso en el que estos organismos pueden provocar fisuras o aprovechar las fracturas preexistentes para ocupar el resto de la superficie, ocasionando nuevas fisuras y/o fracturas (Fig. 4c).

Las piezas en las que se removió parte del talo fueron observadas bajo un microscopio electrónico de barrido (modelo Quanta 200) a abajo vacío. Esto se realizó con el objetivo de poder identificar características particulares de los líquenes a mayor aumento, como así también describir más detalladamente las modificaciones identificadas sobre la roca. Este microscopio cuenta además con un espectrómetro de Rayos-X dispersivo en energías (EDAX, SDD Apolo 40), lo que permitió determinar de manera semicuantitativa elementos de la composición química, tanto de los líquenes como de las superficies rocosas expuestas tras la remoción del talo. En este sentido, se espera que los espectrogramas obtenidos de los análisis de EDAX realizados sobre las alteraciones detectadas, se encuentren asociadas a restos de materia orgánica. La presencia de esta materia orgánica podría corresponder con restos del talo liquénico, sugiriendo que el liquen es la causa de esa alteración. La ausencia de materia orgánica permite suponer que otro factor pudo haber causado la alteración (i.e. abrasión sedimentaria, playas de abrasión, daño térmico, etc.). 

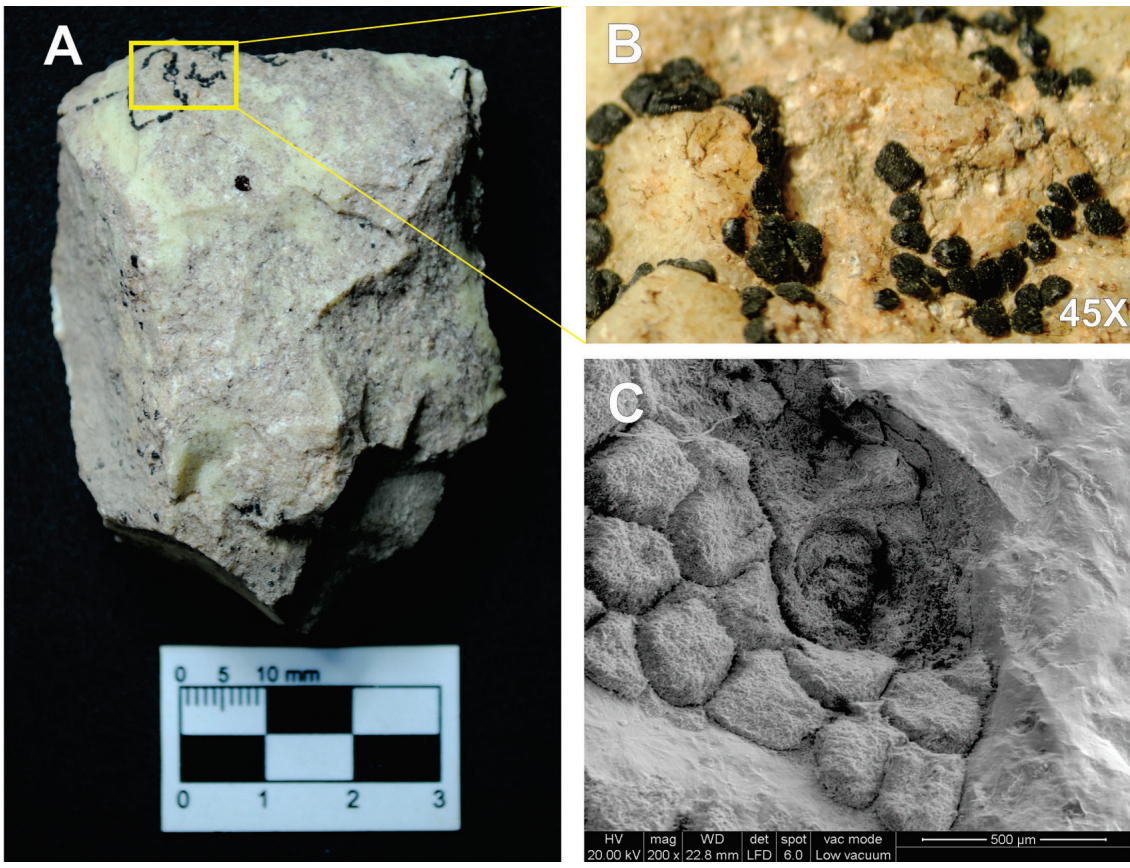

Fig. 3. A: Artefacto F55; B: Liquen de morfología crustosa endolítica observado mediante lupa binocular ( $C$. vorticosa); C: Imagen de MEB de un sector de la pieza donde fue removido el talo en la que se observa una microperforación.

\section{Resultados}

\section{Observaciones a bajos aumentos}

Entre los líquenes presentes, se registraron tres morfotipos. La mayor frecuencia corresponde a los crustosos epilíticos $(\mathrm{n}=11)$, seguidos de crustosos endolíticos $(n=10)$ y foliosos $(n=8)$ (Tabla 1$)$. A partir de esta primera aproximación se procedió a realizar observaciones a mayor aumento, lo que permitió registrar en detalle las morfologías liquénicas y arribar a la determinación de familia en el caso de Physciaceae, de género Acarospora $s p$., (en estos casos los individuos no presentaban estructuras reproductivas o carecían de esporas maduras) y de especie en 16 casos. Las especies determinadas son siete, y las no identificadas, 11. La especie más frecuente es Lecanora wetmorei Śliwa $(\mathrm{n}=7)$, seguida de Carbonea vorticosa (Flörke) Hertel $(n=2)$ y Xanthoria elegans (Link) Th. Fr. $(\mathrm{n}=2)$, presentándose una sola observación para las otras cuatro especies identificadas (Ingvariella bispora (Bagl.) Guderley \& Lumbsch, Rhizocarpon disporum (Nägeli ex Hepp) Müll. Arg., Umbilicaria decusata (Vill.) Zahlbr., Xanthoparmelia hypopsila (Müll. Arg.) Hale (Tabla 1).

En cuanto a la localización en la pieza arqueológica, se registró que la mayoría de los líquenes $(\mathrm{n}=13)$ se disponen en la cara dorsal de los artefactos, seguidos por los que se encuentran sobre superficies de fracturas $(n=6)$ y en la cara ventral de los mismos $(\mathrm{n}=5)$. A su vez, en tres casos el liquen se extiende en ambas caras (dorsal y ventral) y en un solo caso sobre la cara ventral y el talón (Tabla 1). A bajos aumentos se pudo observar también que la mayoría de los líquenes ( $\mathrm{N}=21)$ se localizan sobre superficies modificadas por talla, mientras que en ocho casos se localizan creciendo sobre corteza (Tabla 1).

\section{Observaciones a altos aumentos}

Se observó que la mayoría de los líquenes se encuentran en sectores altos y bajos de la microtopografía de la pieza $(n=13)$, otros se ubican en sectores exclusivamente bajos $(n=11)$, y en menor frecuencia sobre sectores altos $(n=5)$. Se registraron 21 líquenes sobre fracturas o fisuras, 

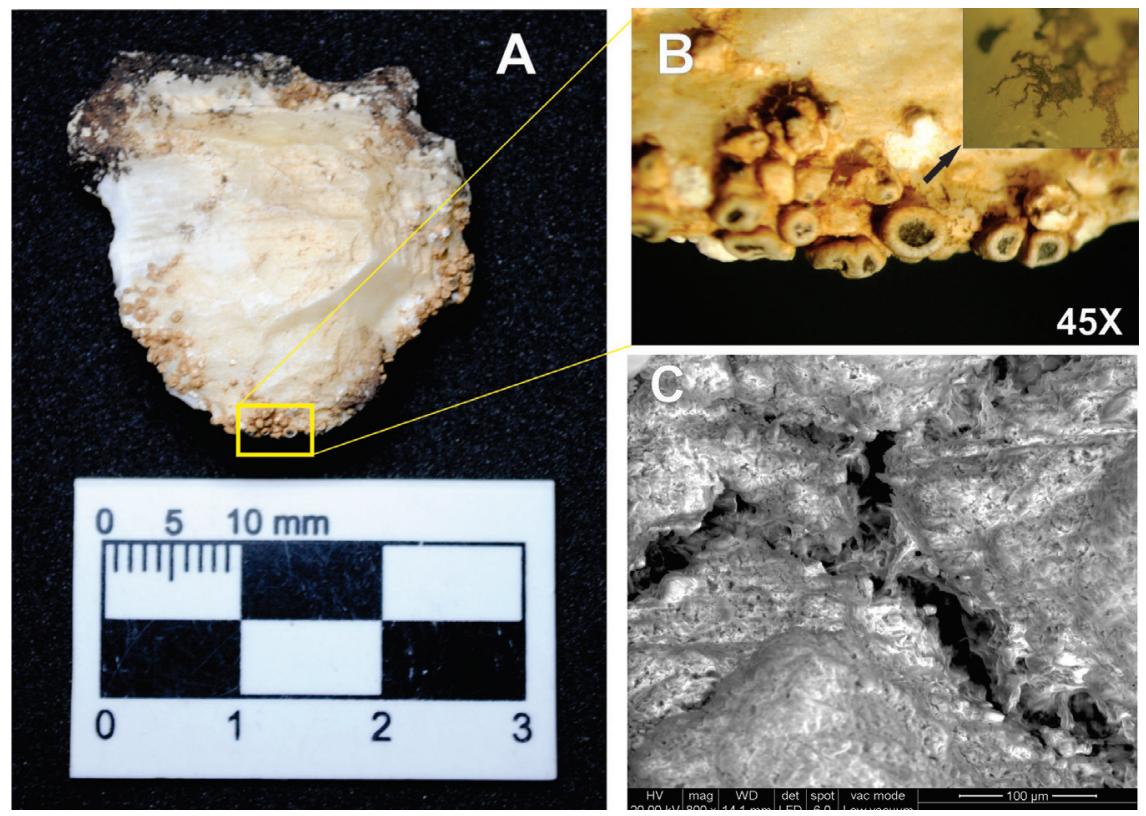

Fig. 4. A: Artefacto D59; B: Liquen de morfología crustosa endolítica observado mediante lupa binocular (L. wetmorei), el recuadro muestra un sector con microfisuraciones internas observado a 63x; C: Imagen de MEB de un sector de la pieza donde fue removido el talo y se observa una microfracturacion superficial.

mientras que ocho se localizaron sobre aristas (Tabla 1). En la mayoría de los casos $(n=23)$ el liquen abarca una posición muy profunda respecto del filo en el campo de observación, y una posición marginal respecto al filo en los cinco restantes.

Se pudieron registrar alteraciones en el $89 \%$ de las observaciones realizadas sobre la superficie rocosa luego de retirar los líquenes. Se constató que las más frecuentes son las microperforaciones y las microfracturaciones internas $(\mathrm{n}=11$ en cada caso), seguidas de la desagregación superficial $(n=10)$ y microfracturaciones superficiales $(n=8)$. Con respecto a los distintos morfotipos, las alteraciones se presentaron en el $100 \%$ de los líquenes crustosos endolíticos; mientras los crustosos epilíticos y foliosos presentaron alteraciones asociadas en el $83 \%$ de los casos.

También hay diferencias en las proporciones de alteraciones observadas por morfotipo. Los líquenes crustosos endolíticos se encuentran asociados principalmentea microperforaciones $(32 \%)$ y microfracturaciones internas $(28 \%)$. Los líquenes del tipo crustoso epilítico, se asocian mayormente con alteraciones de tipo desagregación superficial (43\%), seguidas por microfracturaciones internas $(29 \%)$. Por último, los de tipo folioso se asocian a diversas alteraciones en porcentajes similares $(25 \%)$, pero es llamativa la frecuencia de microfracturaciones superficiales al comparar con los otros morfotipos observados.

\section{$M E B-E D A X$}

La pieza B13 (Fig. 2A) corresponde a una lasca angular de materia prima silícea de color rojo. En cuanto a la presencia de líquenes, fueron identificados sobre su cara dorsal dos tipos: folioso (X. xanthomelanea) y crustoso (no identificado). Las alteraciones que se pudieron detectar incluyen la desagregación superficial de la roca, así como la formación de microperforaciones en sectores bajos de la microtopografía de la pieza (Fig. 2B-C). En los espectrogramas obtenidos mediante EDAX sobre la superficie donde se removieron los líquenes, se identificaron altas concentraciones de carbono y oxigeno; y en menor medida silíceo, aluminio y potasio (Tabla 2). En sectores desprovistos 
Tabla 1. Resultados de las observaciones a bajos y altos aumentos. Disposición de los líquenes sobre el artefacto; líquenes identificados; disposición de líquenes en la microtopografía; y alteraciones observadas.

\begin{tabular}{|c|c|c|c|c|c|c|c|c|c|c|c|c|c|}
\hline & & & BAJOS & S AUMENTOS & & & & LTOS AL & JMEN & NTOS & & & \\
\hline & $\begin{array}{l}\text { Localizaci } \\
\text { en la piez }\end{array}$ & & & Identificación & & $\begin{array}{l}\text { Local } \\
\text { en la }\end{array}$ & $\begin{array}{l}\text { lizac } \\
\text { a pie }\end{array}$ & & & $\begin{array}{l}\text { Alter } \\
\text { aso }\end{array}$ & $\begin{array}{l}\text { acior } \\
\text { ciad }\end{array}$ & & \\
\hline 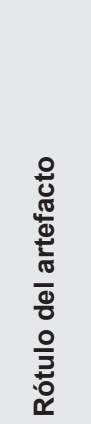 & 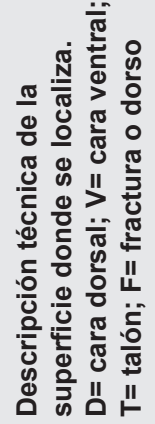 & 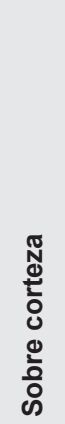 & 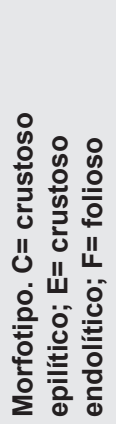 & 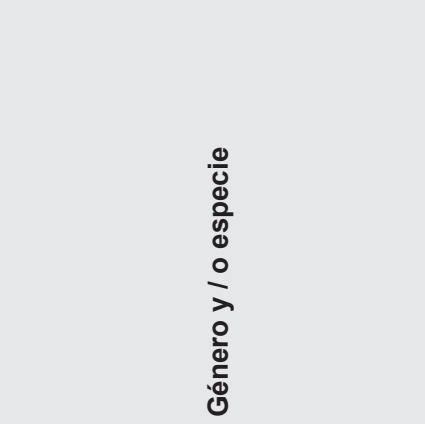 & 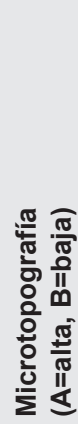 & 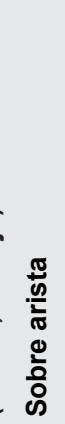 & 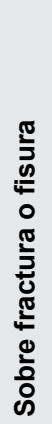 & 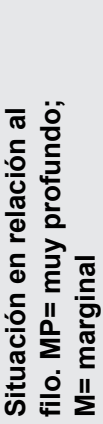 & $\begin{array}{l}\frac{\pi}{0} \\
\frac{0}{\Phi} \\
\Phi \\
\frac{0}{2}\end{array}$ & 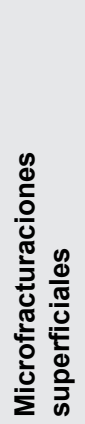 & 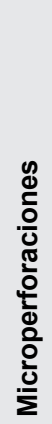 & 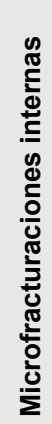 & 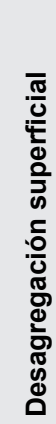 \\
\hline B13 & $D-V$ & 0 & $\mathrm{~F}$ & Xanthoparmelia Xanthomelanea & $\mathrm{H}$ & 1 & 1 & MP & 1 & 0 & 1 & 1 & 1 \\
\hline B13 & $\mathrm{D}$ & 0 & C & indeterminado 1 & $\mathrm{H}$ & 0 & 0 & 0 & - & - & - & - & - \\
\hline B272 & $\mathrm{F}$ & 0 & E & indeterminado 2 & $\mathrm{H}$ & 0 & 1 & M & 1 & 0 & 1 & 0 & 1 \\
\hline $\mathrm{B} 61$ & - & 0 & $\mathrm{~F}$ & Xanthoparmelia hypopsila & $\mathrm{H}$ & 0 & 0 & MP & 1 & 0 & 1 & 1 & 0 \\
\hline $\mathrm{B} 61$ & - & 0 & C & Rhizocarpon disporum & $\mathrm{H}$ & 0 & 0 & MP & 1 & 0 & 1 & 0 & 0 \\
\hline D59 & D-V & 0,1 & $E$ & Lecanora wetmorei & $\mathrm{H}$ & 1 & 1 & MP & 1 & 1 & 1 & 1 & 1 \\
\hline E111 & $\mathrm{F}$ & 0 & E & Lecanora wetmorei & B & 0 & 1 & MP & - & - & - & - & - \\
\hline E116 & $\mathrm{F}$ & 0 & $E$ & Lecanora wetmorei & $\mathrm{H}$ & 0 & 1 & MP & 1 & 1 & 1 & 1 & 0 \\
\hline E145 & D & 1 & $\mathrm{~F}$ & Umbilicaria decussata & A & 0 & 0 & MP & 1 & 0 & 0 & 0 & 1 \\
\hline E166 & D & 0 & $E$ & Lecanora wetmorei & $\mathrm{H}$ & 1 & 1 & MP & 1 & 1 & 1 & 1 & 1 \\
\hline E175 & D & 0,1 & C & indeterminado 3 & B & 0 & 1 & MP & 0 & 0 & 0 & 0 & 0 \\
\hline E269 & $\mathrm{F}$ & 0,1 & C & indeterminado 4 & B & 0 & 1 & MP & 1 & 0 & 0 & 0 & 1 \\
\hline E269 & D & 0 & $\mathrm{~F}$ & Physciaceae & A & 0 & 1 & MP & 0 & 0 & 0 & 0 & 0 \\
\hline E320 & D & 1 & $\mathrm{~F}$ & Xanthoria elegans & $\mathrm{H}$ & 1 & 1 & MP & 1 & 1 & 0 & 0 & 0 \\
\hline E323 & $\mathrm{F}$ & 1 & $\mathrm{~F}$ & Xanthoria elegans & $\mathrm{H}$ & 1 & 1 & MP & 1 & 1 & 0 & 0 & 0 \\
\hline $\mathrm{E} 42$ & D & 0 & C & indeterminado 5 & B & 0 & 1 & MP & - & - & - & - & - \\
\hline $\mathrm{E} 77$ & $\mathrm{~F}$ & 0 & E & Lecanora wetmorei & $\mathrm{H}$ & 0 & 1 & MP & 1 & 0 & 1 & 1 & 1 \\
\hline E81 & D & 0 & $E$ & Lecanora wetmorei & B & 0 & 1 & MP & 1 & 0 & 1 & 1 & 1 \\
\hline E88 & D & 0 & $\mathrm{~F}$ & indeterminado 6 & A & 0 & 1 & M & - & - & - & - & - \\
\hline E88 & V & 0 & C & indeterminado 7 & B & 0 & 1 & M & 1 & 0 & 0 & 1 & 0 \\
\hline E88 & $\mathrm{D}$ & 1 & C & indeterminado 8 & B & 0 & 1 & MP & - & - & - & - & - \\
\hline F55 & D & 0 & E & Carbonea vorticosa & $\mathrm{H}$ & 1 & 1 & MP & 1 & 1 & 1 & 1 & 0 \\
\hline F57 & V-T & 0 & E & Lecanora wetmorei & A & 1 & 0 & MP & - & - & - & - & - \\
\hline G513 & D-V & 0 & C & Ingvariella dispora & B & 0 & 1 & MP & - & - & - & - & - \\
\hline G586 & D & 0 & C & indeterminado 9 & B & 0 & 1 & M & 1 & 0 & 0 & 0 & 1 \\
\hline G863 & D & 0 & $\mathrm{~F}$ & indeterminado 10 & $\mathrm{H}$ & 0 & 0 & MP & - & - & - & - & - \\
\hline G891 & V & 0 & E & Carbonea vorticosa & A & 1 & 1 & M & 1 & 1 & 1 & 1 & 0 \\
\hline J204 & D & 1 & C & Acarospora sp. & B & 0 & 0 & MP & 1 & 1 & 0 & 1 & 1 \\
\hline J204 & $\mathrm{V}$ & 0 & C & indeterminado 11 & B & 0 & 0 & MP & - & - & - & - & - \\
\hline
\end{tabular}


de líquenes se observó una superficie sin las alteraciones mencionadas, aunque se reconocieron rasgos que podrían deberse a abrasión sedimentaria (Lynch et al., 2012, 2015; Lynch, 2016).

La pieza F55 (Fig. 3A) es una lasca angular elaborada sobre un sílex de color amarillo. Sobre esta pieza fue identificado, en la cara dorsal y en el sector proximal, C. vorticosa de morfología crustosa endolítica. El talo se observó creciendo principalmente al interior de fracturas tecnológicas generadas por efecto de la talla de la pieza. Las alteraciones identificadas corresponden principalmente a microperforaciones con un área de ca. 1,6 $\mathrm{mm}^{2}$ y un diámetro máximo de 725 $\mu \mathrm{m}$. También se observaron microfracturaciones superficiales y microfracturaciones internas ocupadas por el talo del liquen que interconecta los apotecios (Fig. 3B-C). El análisis de EDAX realizado en una microperforación permitió observar tres picos significativos correspondientes a oxígeno, silicio y carbono; y otros elementos minoritarios como aluminio, hierro, calcio y potasio (Tabla 2).

La pieza D59 (Fig. 4A) corresponde a una lasca primaria elaborada sobre sílice blanco. La presencia de líquenes fue identificada tanto en la cara dorsal como ventral de la pieza. El liquen identificado corresponde a la especie $L$. wetmorei de morfotipo endolítico. Las alteraciones registradas fueron principalmente desagregación superficial, microperforaciones y en algunos casos microfracturaciones internas, donde luego de la limpieza aún pudo observarse parte remanente del talo (Fig. 4B-C). El análisis de EDAX se realizó sobre una microfracturación superficial, lo que permitió identificar los elementos que se encontraban en su interior. Los más representados corresponden al oxígeno, silíceo, carbono, aluminio y hierro; y concentraciones menores de magnesio, potasio y sodio (Tabla 2).

La pieza J204 (Fig. 5A) es una lasca primaria de sílice marrón. La especie encontrada pertenece al género Acarospora $s p$. de morfotipo crustoso epilitico, y se localiza sobre la cara dorsal de la pieza principalmente en superficies bajas de la microtopografía. Esta especie se encuentra asociada a desagregación superficial de un área aproximadamente circular de 12271,85 $\mu \mathrm{m}^{2}$ que presenta un diámetro de $125 \mu \mathrm{m}$. Aunque se trata de una alteración menos puntual que las microperforaciones, el diámetro máximo de desagregación en este caso (Fig. 5B-C), no supera el de las microperforaciones asociados a la especie C. vorticosa (pieza F55). Cabe aclarar, que el microscopio electrónico utilizado no permite medir la profundidad de estos rasgos, sin embargo a partir de las imágenes tridimensionales pudo definirse que las microperforaciones observadas son relativamente más profundas que la desagregación superficial.

Tabla 2. Resultados de los análisis EDAX. Wt $\%=$ Weight Percentage, Peso porcentual At\%=Atomic Percentage, porcentaje atómico.

\begin{tabular}{|ccccccc|}
\hline $\begin{array}{c}\text { Elementos } \\
\text { Químicos }\end{array}$ & Wt $\%$ & At $\%$ & Wt $\%$ & At $\%$ & Wt \% & At \% \\
C & 53.53 & 61.91 & 18.30 & 28.36 & 20.5 & 30.12 \\
O & 40.58 & 35.24 & 38.27 & 44.51 & 45.04 & 49.66 \\
$\mathrm{Na}$ & - & - & 0.36 & 0.29 & - & - \\
$\mathrm{Mg}$ & - & - & 0.85 & 0.65 & - & - \\
$\mathrm{Al}$ & 1.06 & 0.54 & 12.94 & 8.93 & 4.44 & 2.90 \\
$\mathrm{Si}$ & 4.26 & 2.11 & 21.92 & 14.52 & 24.32 & 15.28 \\
$\mathrm{~K}$ & 0.57 & 0.20 & 2.07 & 0.99 & 0.71 & 0.32 \\
$\mathrm{Fe}$ & - & - & 5.28 & 1.76 & 3.85 & 0.50 \\
$\mathrm{Ca}$ & - & - & - & - & 1.14 & 1.22 \\
Total & 100.00 & 100.00 & 100.00 & 100.00 & 100.00 & 100.00 \\
\hline
\end{tabular}


Bol. Soc. Argent. Bot. 52 (3) 2017
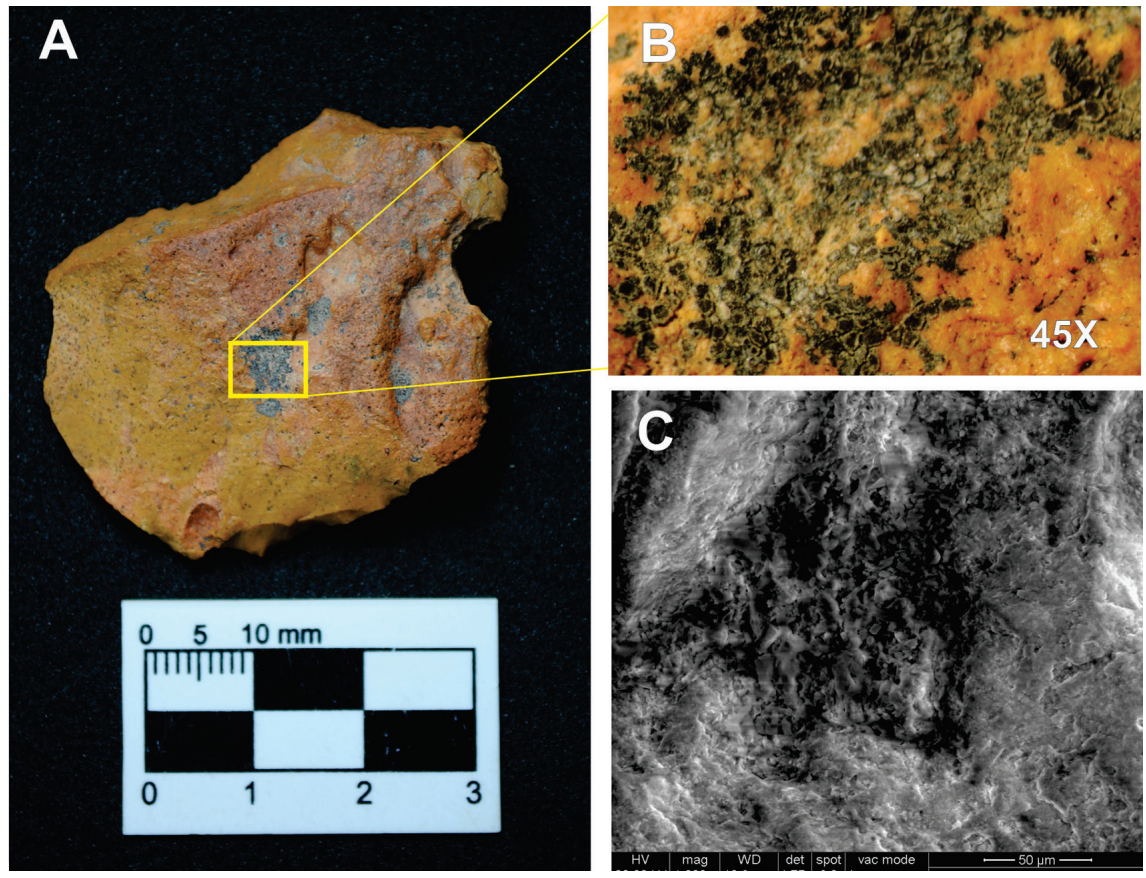

Fig. 5. A: Artefacto J204; B: Liquen de morfología crustosa epilitica observado mediante lupa binocular (Acarospora sp.); C: Imagen de MEB de un sector de la pieza donde fue removido el talo donde se observa desagregación superficial.

\section{Discusión}

Las observaciones realizadas sobre los artefactos de la localidad La Primavera permitieron apreciar una variedad de morfotipos y especies de líquenes presentes.

La diversidad de rocas que conforman la muestra estudiada, podría representar diferentes condiciones para el crecimiento de líquenes sobre ellas. Los sílices, maderas petrificadas y basaltos de textura afanítica, son materiales homogéneos y compactos, mientras que los ocres presentan una textura irregular y moderadamente compacta. De igual modo, las superficies de los artefactos que reservan parte de la corteza natural de la roca, constituyen sustratos diferentes que las superficies modificadas tecnológicamente para la fijación de líquenes. Consideramos entonces que tanto la litología como la presencia o no de corteza son factores pertinentes para seguir indagando en futuros estudios.

En cuanto a las alteraciones registradas en los materiales observados, varias de ellas podrían haber sido bioalteraciones causadas por los líquenes. El hecho de que estos afecten los sustratos rocosos sobre los que se asientan (tanto los naturales como los artificiales) contribuyendo a su deterioro, ha sido abordado por numerosos autores (Seaward, 1997; Silva et al., 1997; Chen \& Blume, 1999; Aptroot \& James, 2002; Videla et al., 2003; Ariño \& Saiz Jimenez, 2004; Bungartz \& Garvie, 2004; St. Clair \& Seaward, 2004; Tratebas, 2004; Rosato, 2010). A favor de esta idea, se registraron alteraciones presentes en la mayoría de las superficies que fueron observadas luego de retirar los talos. En cambio, sobre la superficie no colonizada por estos organismos, la presencia de alteraciones fue considerablemente menor. Asimismo en diversos trabajos, rasgos similares a las alteraciones observadas en esta investigación han sido adjudicados a la acción de líquenes (Prieto Lamas et al., 1995; Ariño et al., 1997; Rosato, 2007; Mohammadi \& Krumbein, 2008).

Incluso aquí se sugieren relaciones en los datos en cuanto a los distintos tipos de alteraciones 
presentes y los morfotipos asociados a ellas. Los resultados obtenidos hasta el momento indican que si bien todos los morfotipos se asocian a algún tipo de bioalteración, es posible diferenciar algunas tendencias. En este sentido, los crustosos epilíticos parecen producir un menor deterioro, afectando solo a la capa superficial del sustrato (principalmente desagregación superficial), mientras que los crustosos endolíticos y foliosos producirían un mayor daño debido a que se desarrollan principalmente hacia el interior del sustrato rocoso (microfracturaciones internas, microperforaciones, microfracturaciones superficiales). Los análisis de EDAX realizados sobre los talos aportan información al respecto. Los elementos que aparecen en mayores concentraciones en el folioso $X$. xanthomelanea son el carbono y el oxígeno (con más del 40\%); mientras que los crustosos endoliticos $(C$. vorticosa y L. wetmorei) presentan además concentraciones de silicio mayores al 20\%. Esta presencia diferencial de silicio, podría deberse a la incorporación de este elemento en el talo y relacionarse con su capacidad para relocalizar los elementos que extrae de la superficie sobre la que se encuentra. Este suceso en el cual partículas de minerales producto del deterioro son atrapadas por el talo fue observado en otras investigaciones (Ascaso \& Wierzchos, 1994; Ariño et al., 1995; Prieto Lamas et al., 1995), lo que sugeriría efectivamente el deterioro activo de las rocas y la participación de los líquenes en esta.

Por otro lado, es necesario remarcar y discutir también que algunas de las alteraciones observadas podrían deberse a otros factores que difieren de la acción de líquenes. Por ejemplo, en otros trabajos se han estudiado agrietamientos y hoyuelos en la superficie de artefactos líticos que son producto de daño térmico. Los rasgos que caracterizan estas alteraciones han sido definidos por otros autores (Mandeville, 1973; Clemente Conte, 1995; Cattáneo et al., 1998; Nami et al., 2000; Terradas \& Gibaja, 2001; Frank, 2012) siendo los más diagnósticos los hoyuelos, escamaciones, rugosidad, agrietamientos, cambios de coloración, lustres y pátinas. Los primeros cuatro indicadores se asocian a daño térmico, es decir, fracturas generadas por la exposición de las piezas al calor. Ocurren en la superficie externa de la pieza, aunque en algunos casos esta queda totalmente destruida y no se puede reconocer la superficie original. Los hoyuelos originados por daño térmico son negativos de forma cónica y generalmente se producen en el cuerpo del artefacto lítico al desprenderse un fragmento denominado cono de desprendimiento (Frank, 2012). La diferencia más inmediata en los hoyuelos dejados por daño térmico y las microperforaciones que podrían estar asociadas a la acción de líquenes está en su tamaño, dado que los primeros pueden verse a simple vista (Cattáneo et al., 1998; Frank, 2012). En cambio el diámetro de las microperforaciones registradas en este trabajo para $C$. vorticosa, es similar a los diámetros medidos por Mohammadi \& Krumbein (2008) causados por la salida del cuerpo fructífero de líquenes endolíticos al exterior del sustrato, y que presentaba variaciones de entre $10 \mu \mathrm{m}$ y 800 $\mu \mathrm{m}$. La detección mediante EDAX de materia orgánica en el interior de las microperforaciones observadas en la pieza F55 sugiere que el rasgo mencionado podría haber sido igualmente formado por la salida del cuerpo fructífero de C. vorticosa desde el interior de la roca.

En cuanto a la profundidad de las microperforaciones, y otras bioalteraciones causadas por líquenes, Rosato (2007) observó que las hifas del liquen crustoso epilítico Caloplaca felipponei Zahlbr penetraban hasta $0,07 \mathrm{~mm}$ en la arenisca sobre la cual se encontraban, mientras que Prieto Lamas et al. (1995) registraron que las hifas de Xanthoria parietina (L.) Th. Fr., Pertusaria leucosora Nyl., Ochrolechia parella (L.) A. Massal., Tephromela atra Fée penetraban el granito hasta $4 \mathrm{~mm}$ de profundidad. En este trabajo la profundidad de penetración de las hifas no fue analizada por ser un procedimiento destructivo que iría en contra de la preservación del material arqueológico.

Otro tipo de bioalteración observada, la desagregación superficial, presenta dimensiones menores a las microperforaciones y podría confundirse con la abrasión sedimentaria, que suele estar presente en diferentes grados en la mayoría de los artefactos líticos arqueológicos (LeviSala, 1993; Mansur-Franchomme, 1999; Pal \& Messineo, 2014; Lynch et al., 2012, 2015; Lynch, 2016). Sin embargo, la desagregación observada muestra cierta profundidad y rugosidad que se diferencia del desgaste que ha sido interpretado como abrasión sedimentaria en otros conjuntos líticos arqueológicos que constituyen sustratos similares (Lynch et al., 2015; Lynch, 2016). 


\section{ConClusión}

Esta primera aproximación al estudio de los procesos de biodeterioro sobre los artefactos líticos recolectados en La Primavera ha permitido una mejor compresión de los efectos que generan la presencia de estos organismos en materiales arqueológicos líticos. Los líquenes pudieron utilizar rastros tecnológicos y fracturas preexistentes, y luego a partir de allí seguir produciendo nuevas alteraciones. Entre las más conspicuas, las microperforaciones implican un mayor daño ya que al producir una apertura de la superficie de la roca permiten que el deterioro también se produzca debajo de la superficie acelerando el proceso. Si bien estas alteraciones en algunos casos son pequeñas e imperceptibles a ojo desnudo, las mismas son el inicio de otros procesos destructivos a mayor escala. En particular, el intento de remover los líquenes para estudio de las piezas arqueológicas, produce el desprendimiento de parte de la superficie a la que se encuentran adheridos con la consiguiente pérdida de material. Estos efectos pueden tener implicancias en las interpretaciones arqueológicas, en el sentido de que su crecimiento podría producir perdida de información en análisis tecnológicos, así como en análisis específicos de la funcionalidad de los artefactos.

Destacamos, sin embargo, que el estudio de los líquenes puede aportar información importante acerca de procesos de formación de sitios arqueológicos, y, en tal sentido es que deben diseñarse protocolos experimentales que permitan seguir trabajando en esta línea dentro de las investigaciones arqueológicas. Es de especial interés desarrollar análisis que busquen evaluar comparativamente las alteraciones producidas por líquenes de otros posibles factores.

\section{Agradecimientos}

Agradecemos al CONICET, LEMIT CIC-PBA y UNLP (Proyecto Promoción a la Investigación N012) por el financiamiento. Al LIMF Servicio de Microscopía Electrónica de Barrido y Microanálisis del Laboratorio de metalúrgica Física (Ing. Gregorio Cusminsky), por el servicio brindado; y a Patrimonio Cultural Santa Cruz; por los permisos de investigación otorgados.

\section{Bibliografía}

ÁLVAREZ, M. R. 2003. Organización tecnológica en el Canal Beagle. El caso de Túnel I (Tierra del Fuego, Argentina). Tesis doctoral. Facultad de filosofía y letras, Universidad de Buenos Aires. Buenos Aires.

APTROOT, A. \& P. W. JAMES. 2002. Monitoring lichens on monuments. In: NIMIS, P. L., C. SCHEIDEGGER \& P. A. WOLSELEY (eds.), Monitoring with Lichens - Monitoring Lichens, pp. 239-253. Kluwer Academic Publishers, Netherlands.

ARINO, X., J. J. ORTEGA-CALVO, A. GOMEZBOLEA, \& C. SAIZ-LIMENEZ. 1995. Lichen colonization of the Roman pavement at Bael0 Claudia (Cadiz, Spain): biodeterioration vs. bioprotection. Sci. Total Environ. 167: 353-363.

ARIÑO, X., J. J. ORTEGA-CALVO, A. GOMEZBOLEA \& C. SÁIZ-JIMÉNEZ. 1997. Lichen colonization of the Roman pavement at Baelo Claudia (Cadiz, Spain): biodeterioration vs. bioprotection. Sci. Total Environ. 167: 353-363.

ARINO, X. \& C. SAIZ-LIMENEZ. 2004. Lichens of Different Mortars at Archaeological Sites in Southern Spain: An Overview. In: L. L. ST. CLAIR \& M. R. D. SEAWARD (eds.), Biodeterioration of Stone Surfaces: Lichens and Biofilms as Weathering Agents of Rocks and Cultural Heritage, pp. 165180. Kluwer Academic Publishers, Dordrecht.

ASCASO, C. \& J. WIERZCHOS. 1994. Structural aspects of the lichen -rock interface using backscattered electron imaging. Bot. Acta 107: 251-256.

ASCHERO, C. A. 1975. Ensayo para una clasificación morfológica de artefactos líticos aplicada a estudios tipológicos comparativos. Informe a CONICET, Buenos Aires. Inédito.

ASCHERO, C. A. 1983. Ensayo para una clasificación morfológica de artefactos líticos. Apéndice A y B. Cátedra de Ergología y Tecnología. Universidad de Buenos Aires. Inédito.

BARRENO, E. \& V. J. RICO. 1984. Sobre la biología de los líquenes. 1. Anatomía, morfología y estructuras vegetativas. An. Biol. 1: 161-195.

BENEDICT, J. 2009. A review of lichenometric dating and its applications to archaeology. Am. Antiq. 74: 143-172.

BJELLAND, T. \& S. EKMAN. 2000. On the occurrence of endolithic hyphae beneath Ophioparma ventosa. Abstracts of the Fourth IAL Symposium, Progress and Problems in Lichenology at the Turn of the Millennium, Barcelona.

BORRAZZO, K. 2011. Tafonomía lítica y pseudoartefactos: el caso de la península El Páramo (Tierra del Fuego, Argentina). Intersecciones antropol. 12: 155-167. 
BÜDEL, B. \& C. SCHEIDEGGER. 2008. Thallus morphology and anatomy. In: Nash, T.H. III (ed.). Lichen biology. Cambridge University Press, Cambridge.

BUNGARTZ, F. \& L. A. GARVIE. 2004. Anatomy of the endolithic Sonoran Desert lichen Verrucaria rubrocincta Breuss: implications for biodeterioration and biomineralization. Lichenologist 36: 55-73.

CABRERA, A. 1971 Fitogeografía de la República Argentina. Bol. Soc. Argent. Bot. 14: 1-42.

CAMUFFO, D. 1995. Physical weathering of stones. Sci. Total Environ. 167: 1-14.

CATtÁneO, R., A. PUPiO, M. VALENTE \& A. BARNA. 1998. Alteración térmica en dos tipos de rocas silíceas: resultados experimentales y aporte de datos para el análisis arqueológico. Relaciones 22-23: 343-361.

CHEN, J. \& H. P. BLUME. 1999. Biotic weathering of rocks by lichens in Antarctica. Chinese Journal of Polar Science 10: 25-32.

CHEN, J., H. P. BLUME \& L. BEYER. 2000. Weathering of rocks induced by lichen colonization - a review. Catena. 39: 121-146.

CLEMENTE CONTE, I. 1995. Sílex y lustre térmico en el Paleolítico Medio ¿Alteración o técnica de talla? El ejemplo de Mediona 1. (Alt. Penedes Barcelona). Actas de Trabalhos de Antropologia y Etnologia 35: 37-43.

DE LA ROSA I. N., M. I. MESSUTI \& L. ŚLIWA. 2012. The Lecanora dispersa group (Lecanoraceae) in Argentina. Lichenologist 44: 101-114.

FRANK, A. D. 2012. Tratamiento y daño térmico de artefactos líticos en los componentes tempranos del sitio Casa del Minero 1, Santa Cruz, Argentina. Chungará 44: 25-37.

GARCÍA, R., L. LABORDA, V. ROSATO \& L. MAGNIN. 2015. Hongos liquenizados hallados sobre restos arqueológicos. Nuevos registros para la provincia de Santa Cruz y un nuevo registro para Argentina. Bol. Soc. Argent. Bot. 50: 3-9.

GARCÍA-ROWE, J. \& C. SAIZ JIMÉNEZ. 1991. Lichens and Bryophytes as agents of deterioration of building materials in Spanish Catdedrals. Int. Biodeterior. Biodegradation 28: 151-163.

GOLUBIC, S., I. FRIEDMANN \& J. SCHNEIDER. 1981. The lithobiontic ecological niche with special reference to microorganisms. J. Sediment. Petrol. 51: 475-478.

GÓMEZ, J. C. \& L. A. MAGNIN. 2008. Cartografía geomorfológica aplicada a un sector de interés arqueológico en el Macizo del Deseado, Santa Cruz (Patagonia Argentina)", Boletín del Instituto de Geografia UNAM 65: 22-37.

HARPER, K. T. \& R. L. PENDLETON. 1993. Cyanobacteria and cyanolichens: can they enhance availability of essential minerals for higher plants? Great Basin Nat. 53: 59-72.

LAMB, I. M. 1958. La vegetación liquénica de los Parques Nacionales Patagónicos. Anales Parques Nac. 7. Buenos Aires.

LEVI-SALA, I. 1993. Use-Wear traces: processes of development and post-depositational alterations. In: ANDERSON, P., S. BEYRIES, M. OTTE \& H. PLISSON (eds), Traces et Functions: les gestes retrouvés, pp. 401-416. ERAUL Editions, Lieja.

LYNCH, V. 2016. Estudio comparativo de la producción y uso de artefactos líticos en el Macizo del Deseado (Santa Cruz, argentina). BAR International Series (2816), Oxford.

LYNCH, V., D. HERMO \& M. ÁLVAREZ. 2012. The use of the form: Functional analysis of Lower Component artefacts from Piedra Museo (Santa Cruz, Argentina). In: L. L. Miotti, M. Salemme, N. Flegenheimer \& T. Goebel (eds.), Current Research in the Pleistocene. Southbound, Late Pleistocene Peopling of Latin America, pp. 133138. Center for the study of the First Americans. Texas University, College Station.

LYNCH, V., M. FERNÁNDEZ \& L. L. MIOTTI. 2015. Estudio experimental sobre la dinámica postdepositacional en rastros de uso: Integridad artefactual del componente 1 de Cueva Maripe (Santa Cruz, Argentina). Magallania 43: 211-229.

MAGNIN, L. 2010. Distribuciones Arqueológicas en la Meseta Central de Santa Cruz. Implicancias Para los Estudios de Uso del Espacio y Movilidad de Sociedades Cazadoras Recolectoras, Tesis doctoral. Facultad de Ciencias Naturales y Museo, Universidad Nacional de La Plata, La Plata.

MAGNIN, L; GARCÍA, R. \& L. LABORDA. 2013. Uso de líquenes para evaluar procesos de formación de superficie. Un caso en Patagonia extraandina, Resúmenes del XVIII Congreso Nacional de Arqueología Argentina, Universidad de La Rioja.

MAGNIN, L., ROSATO, V. \& R. GARCÍA. 2015. Información acerca de los líquenes desarrollados sobre artefactos líticos de superficie. Localidad La Primavera, Santa Cruz (Argentina). Actas del XIX Congreso Nacional de Arqueología Chilena, Ediciones Universidad de Tarapacá y Sociedad Chilena de arqueología.

MANDEVILLE, M. 1973. A consideration of the thermal pretreatment of chert. Plains Anthropologist 18: 177-202.

MANSUR-FRANCHOMME, M. E. 1999. Análisis funcional de instrumental lítico: problemas de formación y deformación de rastros de uso. Actas del XII Congreso Nacional de Arqueología Argentina. La Plata. 
MIOTTI, L., L MARCHIONNI, B. MOSQUERA, D. HERMO \& A. CERASO. 2014. Fechados radiocarbónicos y delimitación temporal de los conjuntos arqueológicos de Cueva Maripe, Santa Cruz (Argentina). Relaciones 39: 509-537.

MOHAMMADI, P. \& W. E. KRUMBEIN. 2008. Biodeterioration of ancient stone materials from the Persepolis monuments (Iran). Aerobiologia 24: 27-33

NAMI, H., G. CATTÁNEO \& M. PUPIO. 2000. Investigaciones experimentales sobre el tratamiento térmico en algunas materias primas de Pampa y Patagonia. An. Inst. Patagon. Ser. Cienc. Nat. 28: 315-329.

NASH, T., C. GRIES \& J. ELIX. 1995. A revision of the Lichen Genus Xanthoparmelia in South America. Bibl. Lichenol. 56: 1-157.

NASH III, T.H., B.D. RYAN, P. DIEDERICH, C. GRIES \& F. BUNGARTZ. 2004. Lichen Flora of the Greater Sonoran Desert Region. Arizona State University. Tampa. Arizona. pp. 742.

ØVSTEDAL D. O. \& R. I. LEWIS-SMITH. 2001. Lichens of Antarctica and South Georgia, A guide for their identification and ecology. Cambridge University Press, Cambridge

PAL, N. \& P. MESSINEO. 2014. Aportes a la interpretación de las actividades llevadas a cabo en sitios superficiales a partir del análisis funcional. Rev. Museo Antr. 7: 79-92.

PRIETO LAMAS, B., M. T. RIVAS BRAE \& B. M. SILVA HERMO. 1995. Colonization by lichens of granite churches in Galicia northwest Spain. Sci. Total Environ. 167: 343-351.

PURDY, B. \& H. BROOKS. 1971. Thermal alteration of silica minerals: An archaeological approach. Science 173:322-325.

ROSATO, V. G. 2007. The lichen Caloplaca felipponei growing on quartz sandstone in Mar del Plata (Argentina): SEM observations. Lilloa. 44: 69-73.

ROSATO, V. G. 2010. Líquenes como posibles agentes de deterioro de areniscas en las ruinas jesuíticas de San Ignacio Miní (provincia de Misiones, Argentina). Materiales de Construcción, 60: 145-153.

SAND, W. 1997. Microbial mechanisms of deterioration of inorganic substrates - a general mechanistic overview. Int. Biodeterior. Biodegrad. 40: 183-190.

SEAWARD, M. R. D. 1997. Major impact made by lichens in biodeterioration processes. Int. Biodeterior. Biodegrad. 40: 269-273.

SEAWARD, M. R. D., C. DIACOBINI, M. R. GIULIANI, \& A. ROCCARDI. 1989. The role of lichens in the biodeterioration of ancient monuments with particular reference to Central Italy. Int. Biodeterior. Biodegrad. 25: 49-55.
SILVA, B., B. PRIETO, T. RIVAS, M. J. SANCHEZBIEZMA, G. PAZ, \& R. CARBALLAL, 1997. Rapid biological colonization of a granitic building by lichens. International Biodeterior. Biodegrad. 40: 263-267.

ŚLIWA, L. 2007. A revision of the Lecanora dispersa complex in North America. Polish Bot. J. 52: 1-70.

SOTO, J. \& M. VAZQUEZ. 2000. Las condiciones climáticas de la Provincia de Santa Cruz. En: GARCÍA \& MAZZONI (ed.), El Gran Libro de la Provincia de Santa Cruz, pp. 98-115. Millenio - Alfa Centro Literario, Madrid.

SOUZA-EGIPSY, V., J., WIERZCHOS, J. V. GARCÍARAMOS, \& A. ASCASO. 2002. Chemical and ultrastructural features of the lichen volcanic/ sedimentary rock interface in a semiarid region (Almería/Spain). Lichenologist 34: 155-167.

ST. CLAIR, L. L. \& M. R. D. SEAWARD. 2004. Biodeterioration of Rock Substrata by Lichens: Progress and Problems. In: L. L. ST. CLAIR \& M. R. D. SEAWARD (eds.), Biodeterioration of Stone Surfaces: Lichens and Biofilms as Weathering Agents of Rocks and Cultural Heritage, pp. 1-8. Kluwer Academic Publishers, Dordrecht.

TERRADAS, X., \& GIBAJA, J. F. 2001. El tratamiento térmico en la producción lítica: el ejemplo del Neolítico Medio catalán. Cypsela 13: 29-56.

TRATEBAS A. M. 2004. Biodeterioration of Prehistoric Rock Art and Issues in Site Preservation. In: L. L. ST. CLAIR \& M. R. D. SEAWARD (eds.), Biodeterioration of Stone Surfaces: Lichens and Biofilms as Weathering Agents of Rocks and Cultural Heritage, pp. 195-228. Kluwer Academic Publishers, Dordrecht.

VIDELA, H. A., P. S GUIAMET \& S. G., GOMEZ de SARAVIA. 2003. Biodeterioro de materiales estructurales de sitios arqueológicos de la civilización maya. Revista Mus La Plata. 44: 1-11.

WIERZCHOS, J. \& C., ASCASO. 1996. Morphological and chemical features of bioweathered granitic biotite induced by lichen activity. Clays Clay Miner. 44: 652-657.

Recibido el 20 de octubre de 2016, aceptado el 30 de diciembre de 2016. 